\title{
COMPARISON OF NUCLEOSYNTHESIS MODELS
}

\author{
O. NAKAMURA \\ Institute of Astronomy, University of Tokyo \\ 2-21-1 Osawa Mitaka, Tokyo 181, Japan
}

Two independent sets of Type II supernova yield models, Woosley \& Weaver (1995) and Nomoto et al. (1997a), are compared with each other by calculation of galactic chemical evolution of 67 stable isotopes from Oxygen through Zinc. Just the same settings of parameters such as star formation rate are used for both sets of models in calculation. Models of Nomoto et al. (1997b) are used for Type Ia supernova. Under this condition, (1) both sets can reproduce solar abundances of $\mathrm{O}, \mathrm{Si}, \mathrm{Cr}$ and $\mathrm{Mn}$ well. (2) Both sets show different paths in all elements at lower metallicity. Because models of Woosley \& Weaver takes into account the metallicity dependence while those of Nomoto et al. offers stars of solar metallicity, it can be understood as the difference of metallicity dependence between both sets. (3) Even the models of Woosley \& Weaver which takes into account the metallicity dependence cannnot completely explain observed abundances of many elements at extremely low metallicities of $[\mathrm{Fe} / \mathrm{H}] \simeq-4$. The figure of $\mathrm{Cr}$ is attached below for exsample.

reference

Woosley \& Weaver,1995

ApJS, 101, 181

Nomoto et al. $1997 \mathrm{a}$ astro-ph, 9706024

Nomoto et al. $1997 \mathrm{~b}$ astro-ph, 9706025

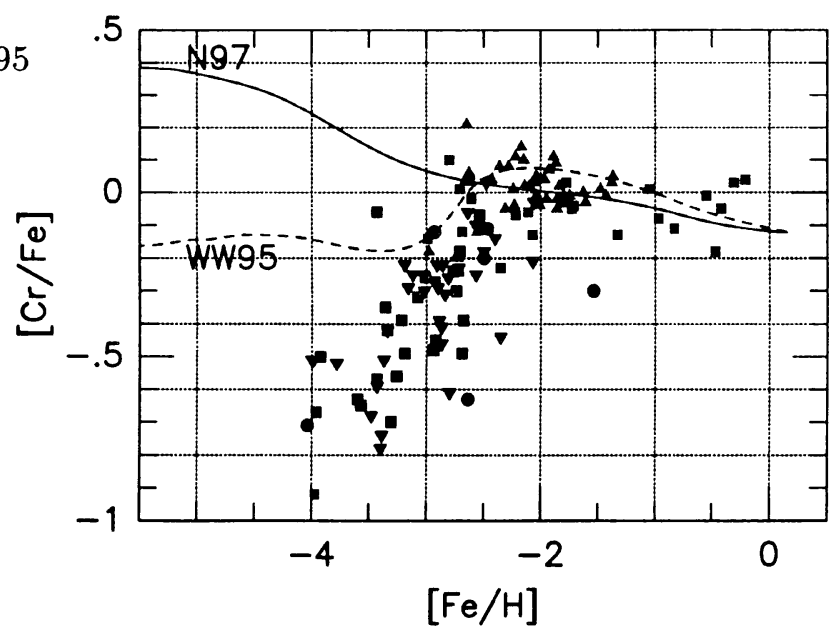

